\title{
Transition from Abelian to Non-Abelian Quantum Liquids in the Second Landau Level
}

\author{
Arkadiusz Wójs \\ TCM Group, Cavendish Laboratory, University of Cambridge, Cambridge CB3 0HE, United Kingdom, and \\ Institute of Physics, Wroclaw University of Technology, Wyb. Wyspiańskiego 27, 50-370 Wroclaw, Poland
}

\begin{abstract}
The search for non-Abelian quantum Hall states in the second Landau level is narrowed to the range of filling factors $7 / 3<\nu_{e}<8 / 3$. In this range, the analysis of energy spectra and correlation functions, calculated including finite width and Landau level mixing, supports the prominent nonAbelian candidates at $\nu_{e}=5 / 2$ (Moore-Read) and 12/5 (Read-Rezayi). Outside of it, the four-flux noninteracting composite fermion model is validated. The borderline $\nu_{e}=7 / 3$ state is adiabatically connected to the Laughlin liquid, but its short-range correlations and charge excitations are different.
\end{abstract}

PACS numbers: 73.43.-f, 71.10.Pm

The incompressible quantum liquids (IQLs) [1] formed in a high magnetic field $B$ by two-dimensional electrons filling various fractions $\nu$ of different Landau levels $\left(\mathrm{LL}_{n}\right.$, $n=0,1, \ldots)$ have been the subject of extensive studies ever since the famous discovery of the fractional quantum Hall (FQH) effect 2]. The most recent storm of interest is motivated by the concept of "topological quantum computation" 3, 4] employing non-Abelian statistics of the Moore-Read "pfaffian" wave function [5, 6] believed to describe the FQH state in a half-filled $\mathrm{LL}_{1}$. Other wave functions with different complexities of braiding statistics have also been proposed [7, 8, 9], elevating the convincing demonstration of non-Abelian statistics in a real physical system to the challenge of greatest current importance.

With the partially filled $\mathrm{LL}_{0}$ successfully described by the composite fermion $(\mathrm{CF})$ theory [10] and with ordered electron phases favored in higher LLs, signatures of nonAbelian statistics are most strongly anticipated in the few known IQLs in $\mathrm{LL}_{1}$. Crucial recent experiments in this LL include confirmation of the quasiparticle (QP) charge of $\frac{1}{4} e$ for the half-filled state at $\nu_{e}=\frac{5}{2}$ [14] and careful measurements of the minute excitation gaps [11, 12, 13]. In theory, the most recent advances have been related to the breaking of particle-hole symmetry in the MooreRead state [15], the role of layer width [16] and LL mixing [17, 18] in real systems, and the nature of QPs [19, 20].

Despite intensive studies, connection of the FQH states in $\operatorname{LL}_{1}\left(\nu_{e}=\frac{5}{2}, \frac{7}{3}, \frac{12}{5}\right.$, or $\left.\frac{11}{5}\right)$ to the few proposed wave functions is not conclusively established, and in some cases it is only tentatively assumed for the lack of other candidates. This is an urgent problem, as the connection of some of these wave functions to the particular conformal field theories is precisely what fuels the anticipation of non-Abelian statistics in nature. Another unresolved puzzle is the discrepancy [13] between the experimental and numerical gaps at $\nu_{e}=\frac{5}{2}$, which undermines the understanding of this state in simple models assuming spin polarization or decoupling from the crystal lattice. On the other hand, a wealth of IQLs found in various systems (electrons or CFs at different fillings of different LLs, in layers of varied width $w$ ) invites a more general question of possible IQLs with arbitrary interactions $V$.
In this Letter we demonstrate (numerically) that nonAbelian IQLs can only emerge in $\mathrm{LL}_{1}$ in the narrow range of filling factors $\frac{7}{3}<\nu_{e}<\frac{8}{3}$. In this range, the known non-Abelian candidates at $\nu_{e}=\frac{5}{2}$ and $\frac{12}{5}$ are closely examined (including finite layer width and LL mixing) and found to have favorable correlation energies and suggestive excitation gaps. Outside of it, the Abelian ground states of noninteracting CFs carrying four flux quanta repeat in both lowest LLs. The borderline $\nu=\frac{1}{3}$ ground state is adiabatically connected to the Laughlin state, but it has a smaller gap, distinct short-range correlations, negative quasihole (QH) energy in narrow layers, and probably different quasielectrons (QEs).

We study spin-polarized $N$-particle systems on a unit sphere with the magnetic monopole of strength $2 Q(h c / e)$ inside 21]. In this geometry, $\mathrm{LL}_{n}$ is a shell of angular momentum $\ell=Q+n$, and different $N$-body wave functions at the same $\nu$ are distinguished by a 'shift' $\gamma$ between LL degeneracy and $\nu^{-1} N$. In contrast to previous exact diagonalization calculations, we do not confine ourselves to the systems with particular $V$, but search for the universality classes $(\nu, \gamma)$ of gapped ground states with arbitrary interactions, attainable in realistic systems (of electrons or CFs) with the suitable choice of $n$ and $w$.

We begin by recalling that many-body dynamics in a degenerate LL is determined by an interaction pseudopotential, defined [21] as the dependence of the pair energy $V$ on the relative angular momentum $m=1,3, \ldots$ Moreover, $V_{m}$ induces particular correlations through its deviation from a straight line over any consecutive $m$ 's [22]. Hence, it is known that low-energy spectra of $V_{m}$ are reproduced by a suitable effective pseudopotential $U_{m}$ with only a few (positive or negative) coefficients.

We have used $U=\left[U_{1}, U_{3}, U_{5}\right]$ in the search for IQLs with (nearly) arbitrary interactions. Higher terms (causing long-range order) were ignored. On the other hand, inclusion of $U_{5}$ was needed for an accurate description of the two lowest (electron or CF) LLs known to host IQL states, while it still allowed for useful graphical representation of the ground state properties in an effectively two-dimensional space of (normalized) parameters $U_{m}$.

We looked at various finite systems $(N, 2 \ell)$ in search of 

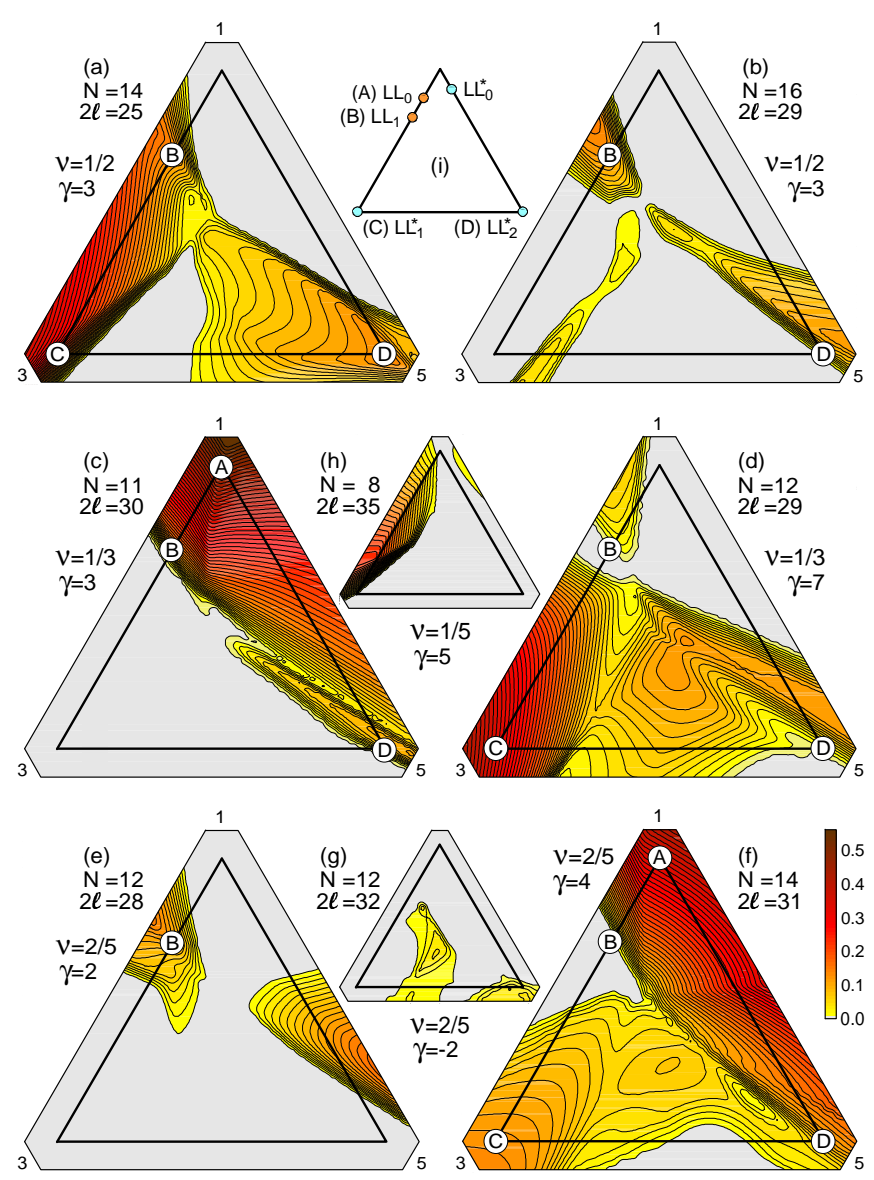

FIG. 1: (color online) Ternary contour plots of the neutral excitation gaps $\Delta$ for $N$ fermions in a partially filled Landau level with shell angular momentum $\ell$, interacting via model pseudopotential $U_{m}$. In each plot, three corners of the big triangles correspond to $U_{m}=\delta_{m, \mu}$ with $\mu=1,3,5$ marked in each corner. Points relevant for the actual interactions in different electron or CF Landau levels are marked on a triangle in frame (i). Different candidate incompressible states are indicated in frames (a)-(h), labeled by filling factors $\nu$ and shifts $\gamma$ assigned to each finite system $(N, 2 \ell)$. For example, the Moore-Read state is marked as (B) in frames (a) and (b).

the series of gapped ground states at $2 \ell=\nu^{-1} N-\gamma$. A few maps of the 'neutral' gap $\Delta$ (gap to the first excited state in the same spectrum) appear in Fig. 1. The IQL candidates are the maxima in $\Delta$ repeating regularly for different $N$. Their location on the map must be compared with the actual pseudopotentials in different LLs, whose approximate positions (A) $-(\mathrm{D})$ are indicated in frame (i).

Let us begin with a half-filled LL represented by $2 \ell=$ $2 N-3$. The maximum near $U_{(B)}=\left[\frac{2}{3}, \frac{1}{3}, 0\right]$ found for each $N$ is relevant for the electrons in $\mathrm{LL}_{1}$, whose actual Coulomb pseudopotential is almost linear between $m=1$ and 5. The maximum coincides with a peak in similar maps (not shown) of the squared overlap of the ground state of $U$ with the exact Moore-Read state, and also with an essentially zero-level minimum of the triplet Hal-

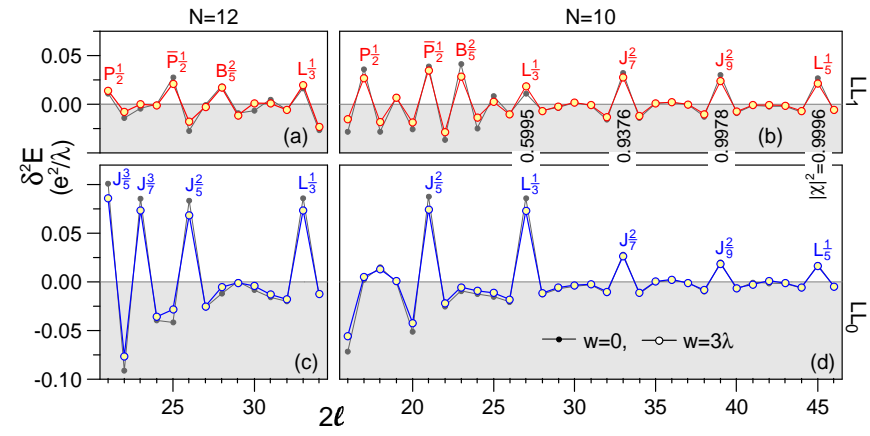

FIG. 2: (color online) Symmetric second-order differences $\delta^{2} E$ of the ground-state energy per particle $E$ for $N=10$ and 12 electrons in $\mathrm{LL}_{0}$ and $\mathrm{LL}_{1}$, as a function of shell angular momentum $\ell$, for layer widths $w=0$ and $3 \lambda$. Candidate incompressible states are marked as ' $\Phi \nu$ ', where $\Phi=\mathrm{P}, \overline{\mathrm{P}}, \mathrm{L}, \mathrm{J}, \mathrm{B}$ denote Moore-Read pfaffian, anti-pfaffian, Laughlin and Jain states, and the state of Ref. [9]. Squared overlaps between the states repeating in both LLs at $\nu \leq \frac{1}{3}$ are indicated.

dane amplitude [23]. This confirms quite definitively the earlier expectations that the Moore-Read ground state indeed occurs for a class of pseudopotentials close to that of $\mathrm{LL}_{1}$ and that its accuracy depends sensitively on the fine-tuning of the leading $V_{m}$ 's, achieved by adjusting the layer width $w$ [16]. Remarkably, it precludes the MooreRead state in other half-filled LLs (e.g., in the second CF LL, called $\mathrm{LL}_{1}^{*}$, with dominant repulsion at $m=3$ [24], where the observed FQH state [25] must be different).

In similar maps for $\nu=\frac{1}{3}$, significant gaps are found in different areas for the series of states with $\gamma=3$ (Laughlin) and 7. The latter was proposed earlier for both $L_{1}$ [22] and $\mathrm{LL}_{1}^{*}$ [26] but, despite numerical evidence for pairing, its wave function remains unknown. It is clear from Fig. 1(c) and (d) that in the vicinity of $U_{(B)}$, relevant for $\mathrm{LL}_{1}$, competition between the $\gamma=3$ and 7 states must be (and will be, in the following) resolved more carefully.

For $\nu=\frac{2}{5}$, Jain's series with $\gamma=4$ correctly represents the ground state in $\mathrm{LL}_{0}$. In $\mathrm{LL}_{1}$, this series competes with two others: the parafermion $\gamma=-2$ state 7] and a recently proposed [9] state with $\gamma=2$. Especially for the latter, Fig. 1(e) appears suggestive of a gap emerging around $U_{(B)}$. The subsequent careful analysis of the competition between these three universality classes is crucial, because the candidate states with $\gamma= \pm 2$ are both non-Abelian, in contrast to the $\gamma=4$ Jain state.

Finally, at $\nu=\frac{1}{5}$, any positive $U=\left[U_{1}, U_{3}, 0\right]$ yields an exact Laughlin state. Fig. 1(h) shows that it is true description of the FQH states in both $\mathrm{LL}_{0}$ and $\mathrm{LL}_{1}$ [27]. On the other hand, its relevance to the FQH effect observed in $\mathrm{LL}_{1}^{*}$ [25] is doubtful (indeed, an alternative series with $\gamma=9$ appears to have more favorable correlations).

Guided by the maps of $\Delta$, pair/triplet amplitudes, and various overlaps we now focus on the FQH states in $\mathrm{LL}_{1}$. In Fig. 2 we seek confirmation of the IQL candidates in cusps of the dependence of the ground-state energy per 


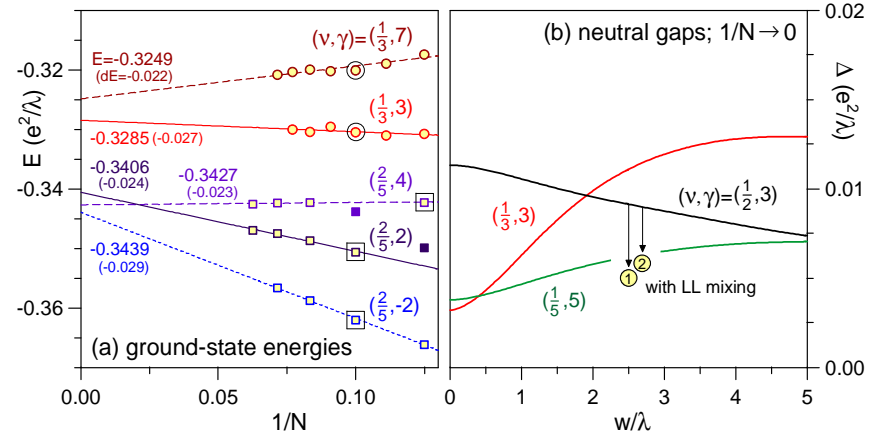

FIG. 3: (color online) (a) Size extrapolation of the groundstate energies per particle $E$ calculated for $N$ electrons in the second Landau level. Competing series of candidate incompressible states with filling factors $\nu=\frac{1}{3}$ and $\frac{2}{5}$ are distinguished by their shifts $\gamma$. Energy corrections $d E$ due to Landau level mixing were obtained for $B=2.6 \mathrm{~T}$ and $N=8$ or 10 , as indicated with large open symbols. (b) Dependence of the extrapolated neutral gaps $\Delta$ on the layer width $w$, for the $\nu=\frac{1}{2}, \frac{1}{3}$, and $\frac{1}{5}$ nondegenerate ground states in the second Landau level. For $\nu=\frac{1}{2}$, circles (1) and (2) show gap reduction due to Landau level mixing relevant for the experimental Refs. [13] and [11], respectively.

particle $E$ on the LL degeneracy. The cusps are most pronounced in the plots of $\delta^{2} E_{2 \ell}=E_{2 \ell-1}+E_{2 \ell+1}-2 E_{2 \ell}$. For an IQL, its (positive) value gives the QP gap $\tilde{\Delta}$ times the number of QPs created per flux quantum.

Besides complementing the maps of Fig. 1 1 in the identification of the universality classes of particular IQLs, Fig. 2 reveals a simple connection between statistics and filling factor in $\mathrm{LL}_{1}$. At $\nu<\frac{1}{3}$ the same Laughlin/Jain IQLs occur in $\mathrm{LL}_{1}$ and $\mathrm{LL}_{0}$, with high overlaps and similar gaps. This similarity, earlier pointed out in Ref. 27], is caused by sufficiently high $V_{1}$ and similar $V_{m \geq 3}$ in both LLs. It validates the noninteracting $C F$ model [10] with four flux quanta attached to each electron in $\mathrm{LL}_{1}$.

In contrast, at $\nu>\frac{1}{3}$ the Jain sequence of $\mathrm{LL}_{0}$ is replaced in $L_{1}$ by a different set of IQLs, including the pfaffian and anti-pfaffian pair at $\nu=\frac{1}{2}$ and, apparently, a different $\nu=\frac{2}{5}$ state with $\gamma=2$ (Fig. 2 shows no signatures of the parafermion $\nu=\frac{2}{5}$ series with $\left.\gamma=-2[7]\right)$. The breakdown of the noninteracting two-flux $\mathrm{CF}$ model in $\mathrm{LL}_{1}$ opens, exclusively at $\frac{1}{3}<\nu<\frac{2}{3}$, a possibility for other IQLs, including several suggested more exotic states with various non-Abelian statistics of their QPs.

For the borderline $\nu=\frac{1}{3}$ state, separating the Abelian from (possibly) non-Abelian states, our calculation reinforces the theory of Ref. 27]. This state has a moderate overlap with the Laughlin ground state of $\mathrm{LL}_{0}$, despite falling into the same class of $\gamma=3$. It also has a smaller gap than the other Laughlin/Jain states in $\mathrm{LL}_{0}$ or $\mathrm{LL}_{1}$.

Having narrowed the search for non-Abelian IQLs in $\mathrm{LL}_{1}$ to the range of $\frac{1}{3}<\nu<\frac{2}{3}$, let us now decide more conclusively between the competing candidate wave functions for the particular filling factors. For $\nu=\frac{1}{2}$, the issues of the interplay of pfaffian and anti-pfaffian states [15] and of spin polarization [28] were already discussed. In Fig. 3(a) we compare the ground-state energies per particle $E$ of three known candidates for $\nu=\frac{2}{5}$ (we used $N \leq 16$ and $w=0$; results for $w>0$ are similar; energies $E$ are rescaled by $\sqrt{2 Q \nu / N}$ to ensure equal units $e^{2} / \lambda$ for each $\gamma$ ). In the extrapolation we only used the open squares, discarding one state aliased with the antipfaffian and one apparently suffering from the small size.

Although Fig. 3(a) suggests that the ground state at $\nu=\frac{2}{5}$ might have $\gamma=-2$ (despite the lack of significant gaps in Figs. (1 and 2), the extrapolated energies of all three competing states are indecisively close. Hence, comparison of their susceptibility to LL mixing is crucial. We estimated the appropriate energy correction $d E$ by including in the diagonalization additional states involving a single cyclotron excitation [17]. For each $\gamma$, the value of $d E$ was calculated for $N \leq 10$ (it was also checked that $d E$ is less $N$-dependent than $E)$. The assumed Coulombto-cyclotron energy ratio $\beta \equiv\left(e^{2} / \lambda\right) /\left(\hbar \omega_{c}\right)=1.56$ corresponds to $B=2.6 \mathrm{~T}$. Clearly, in addition to having the lowest $E$, the $\gamma=-2$ parafermion state also has the largest $|d E|$, and therefore it is convincingly predicted to define the universality class of the $\nu=\frac{2}{5}$ ground state in $L_{1}$. This is important because this state is the only known candidate IQL [4] whose braiding rules are sufficiently complex to allow quantum computation.

Fig. 3(a) presents also the energies for $\nu=\frac{1}{3}$. Here, the $\gamma=7$ series is firmly ruled out in favor of the $\gamma=3$ states (adiabatically connected to the Laughlin state).

In Fig. 3(b) we plot the extrapolated neutral gaps $\Delta(w)$ for several IQLs in $L_{1}$. Unlike in $\mathrm{LL}_{0}$ and in agreement with the experiments [11, 12], the gaps at $\nu=\frac{1}{2}$, $\frac{1}{3}, \frac{1}{5}$ are all similar, and the gap at $\nu=\frac{2}{5}$ is much smaller (not shown). Understanding that $\Delta$ includes the $\mathrm{QE}-\mathrm{QH}$ attraction, it is nevertheless noteworthy that incorporating an earlier estimate of gap reduction due to LL mixing at $\nu=\frac{1}{2}$ [17] gives a surprisingly good agreement with experiments in the high mobility limit: for Refs. [11, 12], $w / \lambda=2.7$ and $\beta=1.1$, yielding the $35 \%$ reduction and $\Delta=0.006 e^{2} / \lambda$; for Ref. [13], $w / \lambda=2.5$ and $\beta=1.7$, yielding the $45 \%$ reduction and $\Delta=0.005 e^{2} / \lambda$.

Let us now examine more closely the crossover $\nu=\frac{1}{3}$ ground states in $L_{1}$. For $N \leq 12$, their squared overlaps with the Laughlin state are merely $\sim 50 \%$. This reflects quite different correlations (e.g., for $N=12$ and $w=3 \lambda$, the pair amplitudes at $m=1,3,5,7$ are $0.02,0.13,0.17$, 0.10 in $\mathrm{LL}_{1}$, as opposed to $0.00,0.18,0.13,0.09$ in $\mathrm{LL}_{0}$ ). The magneto-roton band is also absent in the spectra of $\mathrm{LL}_{1}$. The low-energy states resembling Laughlin QEs and QHs are found at $2 \ell=3 N-3 \mp 1$, but they are not generally the lowest states in their spectra. For example, the ground state at $2 \ell=3 N-4$ has angular momentum $L=2, \frac{3}{2}, 1, \frac{1}{2}, 0$ for $N=8, \ldots, 12\left(\right.$ instead of $\left.L=\frac{1}{2} N\right)$.

Although we have checked that the eigenstates adiabatically connected to the Laughlin QPs become the low- 


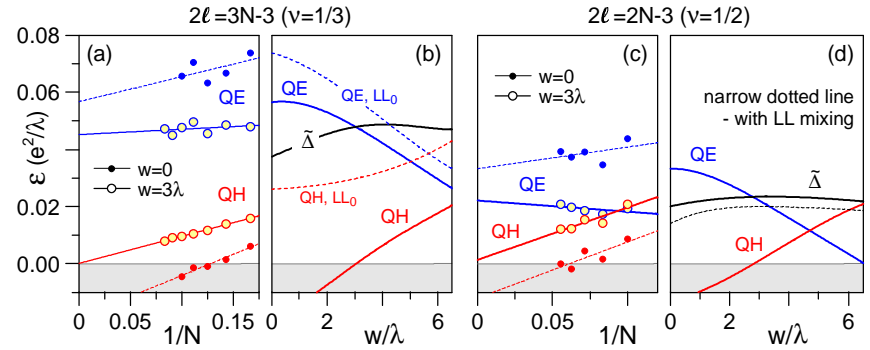

FIG. 4: (color online) Quasiparticle energies $\varepsilon$ at the filling factors $\nu=\frac{1}{3}$ and $\frac{1}{2}$ in the second Landau level: (a,c) Size extrapolation of the $N$-electron results for the layer widths $w=0$ and $3 \lambda$. (b,d) Extrapolated quasielectron and quasihole energies, and their sum $\tilde{\Delta}$, as a function of width $w$. In (b), curves for the lowest Landau level are added for comparison. In $(\mathrm{d})$, dotted line includes Landau level mixing $(B=2.6 \mathrm{~T})$.

est states under bias, their joint energy $\tilde{\Delta}=\varepsilon_{\mathrm{QE}}+\varepsilon_{\mathrm{QH}}$ is unreasonably high. Fig. $4(\mathrm{a}, \mathrm{b})$ shows the size extrapolation of $\mathrm{QE}$ and $\mathrm{QH}$ energies $\varepsilon$, and width dependences of the extrapolated $\varepsilon$ 's. Remarkably, $\varepsilon_{\mathrm{QH}}>0$ requires finite width $w>3 \lambda$. Negative $\mathrm{QH}$ energies in narrow layers are also found in other states in $\mathrm{LL}_{1}$ identified in Fig. 2(b). For instance, $\varepsilon=\frac{1}{2}\left(E_{2 \ell \pm 1}-E_{2 \ell}\right)$ plus the size corrections [29] are plotted in Fig. 4(c,d) for $\nu=\frac{1}{2}$.

Note that the gap $\tilde{\Delta}$ at $\nu=\frac{1}{2}$ is not immediately weakened in wider wells (in contrast to Ref. [29] where only data for $N=10$ and 14 was used for $w>0$ ). The initial gap enhancement in wider wells is even more pronounced with the inclusion of LL mixing (here, the corrections $d E$ were calculated for $N \leq 14$ at $2 \ell=2 N-3$ and $2 N-3 \pm 1$ ). While the reasonable match between the experimental gaps and $\Delta$ (rather than $\tilde{\Delta})$ at $\nu=\frac{1}{2}$ remains a mystery, the difference between the latter two is well understood as the QE-QH atraction [29]. However, the difference between $\Delta$ and $\tilde{\Delta}$ at $\nu=\frac{1}{3}$ is too large to be explained in this way. Instead, Laughlin QEs are unlikely to be the elementary negative carriers in $L_{2}$.

In conclusion, we have found the range of filling factors $\frac{1}{3}<\nu<\frac{2}{3}$ in $\mathrm{LL}_{1}$ in which the emergence of non-Abelian statistics is possible. Inside this range, our calculations including finite layer width and LL mixing demonstrate that the spin-polarized ground states at $\nu=\frac{1}{2}$ and $\frac{2}{5}$ are described by the "pfaffian" and "parafermion" wave functions (both non-Abelian). Outside, the Jain states of noninteracting CFs repeat in both lowest LLs, precluding more exotic phases. The borderline $\nu=\frac{1}{3}$ state is adiabatically connected to the Laughlin liquid but has a smaller gap and distinct excitations.

The author thanks Gunnar Möller, Nigel Cooper, and Guillaume Gervais for many insightful comments, and acknowledges support from EU under the Marie Curie Intra-European Grant No. PIEF-GA-2008-221701 and from the Polish MNiSW under grant N202-071-32/1513.
[1] R. B. Laughlin, Phys. Rev. Lett. 50, 1395 (1983).

[2] D. C. Tsui, H. L. Störmer, and A. C. Gossard, Phys. Rev. Lett. 48, 1559 (1982).

[3] A. Yu. Kitaev, Ann. Phys. (N.Y.) 303, 1 (2003); S. Das Sarma, M. Freedman, and C. Nayak, Phys. Rev. Lett. 94, 166802 (2005).

[4] C. Nayak, S. H. Simon, A. Stern, M. Freedman, and S. Das Sarma, Rev. Mod. Phys. 80, 1083 (2008).

[5] G. Moore and N. Read, Nucl. Phys. B 360, 362 (1991); M. Greiter, X.-G. Wen, and F. Wilczek, Phys. Rev. Lett. 66, 3205 (1991).

[6] Y. Tserkovnyak and S. H. Simon, Phys. Rev. Lett. 90, 016802 (2003).

[7] N. Read and E. Rezayi, Phys. Rev. B 59, 8084 (1999).

[8] G. Möller and S. H. Simon, Phys. Rev. B 77, 075319 (2008).

[9] P. Bonderson and J. K. Slingerland, Phys. Rev. B 78, 125323 (2008); G. Möller et al., in preparation.

[10] J. K. Jain, Phys. Rev. Lett. 63, 199 (1989).

[11] W. Pan, J. S. Xia, H. L. Stormer, D. C. Tsui, C. Vicente, E. D. Adams, N. S. Sullivan, L. N. Pfeiffer, K. W. Baldwin, and K. W. West, Phys. Rev. B 77, 075307 (2008).

[12] H. C. Choi, W. Kang, S. Das Sarma, L. N. Pfeiffer, and K. W. West, Phys. Rev. B 77, 081301(R) (2008).

[13] C. R. Dean, B. A. Piot, P. Hayden, S. Das Sarma, G. Gervais, L. N. Pfeiffer, and K. W. West, Phys. Rev. Lett. 100, 146803 (2008).

[14] M. Dolev, M. Heiblum, V. Umansky, Ady Stern, and D. Mahalu, Nature 452, 829 (2008).

[15] M. Levin, B. I. Halperin, and B. Rosenow, Phys. Rev. Lett. 99, 236806 (2007); S.-S. Lee, S. Ryu, C. Nayak, and M. P. A. Fisher, ibid. 99, 236807 (2007); M. R. Peterson, K. Park, and S. Das Sarma, ibid. 101, 156803 (2008).

[16] M. R. Peterson, Th. Jolicoeur, and S. Das Sarma, Phys. Rev. Lett. 101, 016807 (2008).

[17] A. Wójs and J. J. Quinn, Phys. Rev. B 74, 235319 (2006).

[18] S. H. Simon, Phys. Rev. Lett. 100, 116803 (2008).

[19] C. Töke, N. Regnault, and J. K. Jain, Phys. Rev. Lett. 98, 036806 (2007).

[20] B. A. Bernevig and F. D. M. Haldane, Phys. Rev. Lett. 100, 246802 (2008); arXiv:cond-mat/0810.2366.

[21] F. D. M. Haldane, Phys. Rev. Lett. 51, 605 (1983).

[22] A. Wójs, Phys. Rev. B 63, 125312 (2001).

[23] A. Wójs and J. J. Quinn, Phys. Rev. B 71, 045324 (2005).

[24] S.-Y. Lee, V. W. Scarola, and J. K. Jain, Phys. Rev. Lett. 87, 256803 (2001).

[25] W. Pan, H. L. Störmer, D. C. Tsui, L. N. Pfeiffer, K. W. Baldwin, and K. W. West, Phys. Rev. Lett. 90, 016801 (2003).

[26] A. Wójs, D. Wodziński, and J. J. Quinn, Phys. Rev. B 74, 035315 (2006).

[27] N. d'Ambrumenil and A. M. Reynolds, J. Phys. C 21, 119 (1988).

[28] R. H. Morf, Phys. Rev. Lett. 80, 1505 (1998).

[29] R. H. Morf, N. d'Ambrumenil, and S. Das Sarma, Phys. Rev. B 66, 075408 (2002). 